\title{
DESENVOLVIMENTO DE UM APLICATIVO DE REALIDADE AUMENTADA PARA APLICAÇÃO NO CURSO DE MANUTENÇÃO AEROESPACIAL
}

\author{
DOI: 10.37702/2175-957X.COBENGE.2021.3475
}

Ariane de Novais Bezerra Rodrigues - ariane.bezerra@fatec.sp.gov.br

Faculdade de Tecnologia de São José dos Campos

Rua Kiyoshi Enomoto 775

12235-831 - São José Dos Campos - SP

Jackson Paul Matsuura - jackson@ita.br

Instituto Tecnológico de Aeronáutica

Cândido Marciano Leite 105

12245-486 - São José dos Campos - SP

Resumo: A tecnologia de realidade aumentada (AR) tem sido muito procurada e sendo alvo de pesquisas pela sua facilidade de uso e da interação em tempo real que existe entre o mundo virtual e o seu usuário. Com uma utilização de modelos tridimensionais (por intermédio de um smartphone ou tablet) sendo sobrepostos em ambientes reais, a realidade aumentada é bem avaliada e adequada para ser aplicada no setor educacional auxiliando cada vez mais o ensino-aprendizagem, aumentando mais o interesse e o aprendizado dos alunos. Este artigo consiste na apresentação do desenvolvimento de um aplicativo compatível com o sistema operacional Android (mais usual entre os alunos e professores), auxiliando nas aulas de manutenção aeroespacial tanto em aulas presenciais quanto em aulas remotas, tendo como objeto de estudo um atuador de trem de pouso, podendo ser utilizado em: explicação de montagem e desmontagem do atuador; demonstração de manutenção preventiva e corretiva; aplicação de exercícios; extraclasse.

Palavras-chave: Realidade aumentada. Engenharia. Educação. Manutenção. Aeronaves. 


\section{(C) COBENGE \\ 28 a 30 de SETEMBRO \\ DESENVOLVIMENTO DE UM APLICATIVO DE REALIDADE AUMENTADA PARA APLICAÇÃO NO CURSO DE MANUTENÇÃO AEROESPACIAL}

\section{INTRODUÇÃO}

Realidade aumentada e a realidade virtual são tecnologias interativas que cresceram substancialmente na década de 90 , pelo fato de que o avanço tecnológico viabiliza a possibilidade de execução computacional gráfica interativa em tempo real (KIRNER; SISCOUTTO, 2007, p.4). Tanto a realidade virtual quanto a realidade aumentada, podem ser utilizadas em aplicações individuais e/ou em aplicações coletivas, podendo ser locais ou remotas proporcionando experiências colaborativas com essas tecnologias (BILLINGHURST; KATO, 1999). Muitas empresas já adotaram o uso de realidade aumentada no seu processo de manutenção, treinamento, no setor de marketing, entre outras aplicações, como por exemplo a Boeing. A empresa já utiliza essa tecnologia em manutenção no sistema elétrico das aeronaves, e alega que com uso de realidade aumentada houve um aumento de $90 \%$ na qualidade dos serviços em comparação do uso de materiais contendo informações bidimensionais, por possibilitar a visualização da fiação elétrica em toda a profundidade na fuselagem da aeronave sem dificuldade, e acesso às instruções sem o uso das mãos (Boeing, 2018).

Tendo em vista que a realidade aumentada vem sendo considerada uma tendência no mundo corporativo, e cada vez mais um objeto de estudo, e considerando que os métodos tradicionais de ensino estão ficando cada vez mais defasados pelo fato de que as novas gerações estão crescendo totalmente em uma era digital, a realidade aumentada se torna importante na educação (e consequentemente no mercado de trabalho), facilitando a compreensão do aluno no assunto ministrado, e oferecendo uma melhor comunicação entre professor e aluno (DOMINGOS; DAUM; SILVA; CABRAL, 2012). Segundo Ronald T. Azuma (1997, p.356), "AR permite ao utilizador ver o mundo real, com objetos virtuais sobrepostos ou compostos com o mundo real". Em busca de mais autonomia no ensinoaprendizagem e na execução de serviços, a realidade aumentada é uma tecnologia que está crescendo na área educacional, por conter um uso simplificado, e pela utilização de modelos tridimensionais de alta qualidade, despertando mais o interesse dos alunos pelo uso e pelos assuntos propostos, e aumentando mais a confiança do professor (DADALD, 2015).

Levando esses conceitos em consideração, neste artigo será apresentado o desenvolvimento e o estudo da aplicabilidade de um aplicativo de realidade aumentada no ensino de manutenção aeroespacial, e, para isso, será realizada uma revisão bibliográfica qualitativa com seleção de artigos em conformidade com o assunto proposto.

\section{REFERENCIAL TEÓRICO}

Diversas pesquisas e trabalhos foram realizados tendo como base a aplicação de realidade aumentada no ensino de disciplinas expositivas em escolas e faculdades, para estimular mais a autonomia dos alunos por meio de atividades interativas auxiliando no ensino-aprendizagem e aumentando mais o interesse dos alunos com os assuntos propostos pelo professor, que por vezes tenham sido considerados assuntos de difícil assimilação.

Na pesquisa realizada por Domingos (2012, p.1), ele assegura que a interatividade que a realidade aumentada proporciona no processo do ensino-aprendizagem, utilizando 
de objetos de aprendizagem (OA), se torna um processo mais descontraído, colaborando assim para o envolvimento do aluno nas atividades propostas em sala de aula e que 0 objeto de aprendizagem se torna um estimulante ao desenvolvimento da autonomia do aluno e, pelo fato de ser apresentado de uma forma interativa, pode potencializar muito mais este desenvolvimento dos alunos.

Na pesquisa realizada por Júnior (2017, p.53), a realidade aumentada se apresenta como ferramenta extremamente válida em prover uma interação capaz de atrair a atenção dos alunos para um aprendizado, utilizando de elementos lúdicos atingindo os objetivos com eficiência e eficácia.

Em uma pesquisa realizada por Silva (2011), para entender melhor as diversas áreas de ensino em que a realidade virtual aumentada vem sendo aplicada, é citado de que Byrne em 1996 demonstrou que os estudantes do ensino médio tinham uma retenção de informações muito superior aos estudantes que obtiveram tais informações através de outros meios tecnológicos, por ter a experiência de interagir visualmente e com manuseio de moléculas (experiências de química), pois a interação é realizada naturalmente como se o objeto fosse real, um modelo de interação a qual o usuário já está acostumado com o objeto de aprendizagem.

Segundo Bazarov $(2017$, p.6), a realidade aumentada é uma tecnologia economicamente eficaz, que fornece aos estudantes uma forma de aprendizagem mais atrativa e com conteúdo mais demonstrativo do que o material didático em papel. Assim, os estudantes se sentem mais à vontade para trabalhar com os componentes em 3D, pois é uma tecnologia intuitiva ao qual não necessita de um estudo adicional para o seu uso.

Algumas faculdades (CABERO-ALMENARA; FERNÁNDEZ-BATANERO; BARROSO-OSUNA, 2019), escolas técnicas (BACCA, 2015), e até mesmo escolas de ensino fundamental (MADANIPOUR; COHRSSEN, 2019), e médio (PETROV; ATANASOVA, 2020), já adotaram esse tipo de tecnologia para melhorar mais a compreensão dos alunos e ter a flexibilidade do uso da realidade aumentada de uma forma remota, com o uso de multiplayer, podendo avaliar o avanço dos alunos e sua perfeita assimilação do assunto. Como mencionado, a tecnologia de imersão visual já está sendo aplicada em muitas áreas, mas tem pouca aplicação no ensino de manutenção de peças, componentes aeroespaciais, considerando que é primordial que a manutenção de tal área seja realizada corretamente, evitando possíveis acidentes catastróficos, colocando a vida de muitos em risco.

\section{METODOLOGIA}

Estudos e pesquisas foram necessários para: a determinação do software a ser utilizado no desenvolvimento do aplicativo, para a definição do objeto de aprendizagem para as aulas de manutenção aeroespacial e para entender melhor as dificuldades enfrentadas pelos professores e pelos alunos em sala de aula, para que o aplicativo desenvolvido realmente auxilie no ensino-aprendizagem.

Para o desenvolvimento do aplicativo com realidade aumentada, foi preciso algumas pesquisas de plataformas que oferecem alguns recursos como uma relativa facilidade de programação, considerável quantidade de recursos, componentes pré-programados e a viabilidade de desenvolvimento do aplicativo para outros sistemas operacionais, como o Android e IOS. A plataforma mais adequada que supriu todas as necessidades para o desenvolvimento do aplicativo foi o Unity 3D, ao qual é uma plataforma muito utilizada para o desenvolvimento de aplicativos para treinamentos de diversas áreas, jogos e é uma plataforma de desenvolvimento gratuita, ou seja, sem cobranças de direitos de utilização 
desde que o aplicativo seja criado sem fins lucrativos, conjecturado em contrato (UNITY, [s.d.]).

\subsection{Objeto de Aprendizagem}

Nas aulas de manutenção aeroespacial, são realizados treinamentos, exercícios práticos e teóricos com muitos dispositivos e peças de uma aeronave, e um desses objetos é o atuador do trem de pouso. A disposição do trem de pouso determina a transferência de carga para a estrutura, estabilidade e controle da aeronave no solo (KAWAKAME; MARTINS, 2016) (Figura 1), se tornando muito importante sua montagem e manutenção correta (POLITECNICO DI MILANO, 2004).

Figura 1: Estabilidade do trem de pouso

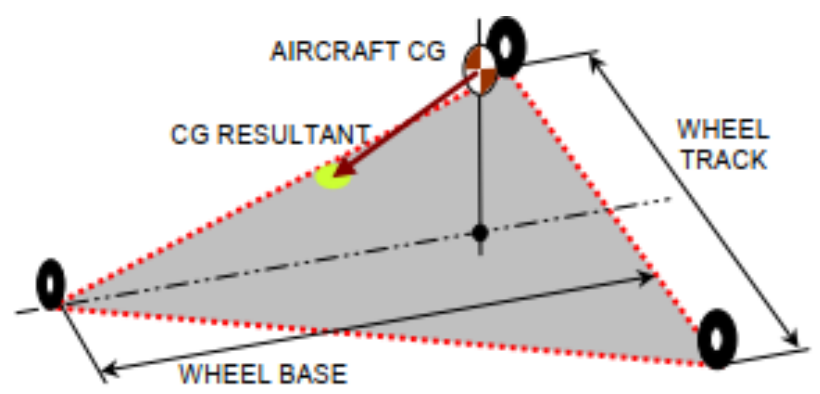

Fonte: Politecnico di Milano

Cada projeto de aeronave tem um trem de pouso diferente. Dependendo das necessidades de estabilidade, de qual modelo de aeronave será construída (aeronave de carga, de transporte, entre outros), requer um trem de pouso especial para cada uma delas. Considerando a quantidade de atuadores de trem de pouso existentes, foi necessário pesquisas e reuniões com professores da aula de manutenção aeroespacial, para a definição de um atuador que atendesse os requisitos das aulas e de fácil montagem e desmontagem, para que as explicações não se tornassem complexas pela quantidade de peças existentes e montagens difíceis de serem realizadas.

O atuador escolhido (atuador de trem de pouso "P/N 200-0300") é constituído por 77 peças, considerando componentes como anéis de vedação, anéis $O$-ring, retentores, molas e peças manufaturadas (Figura 2). 
Figura 2: Atuador do trem de pouso "P/N 200-0300"

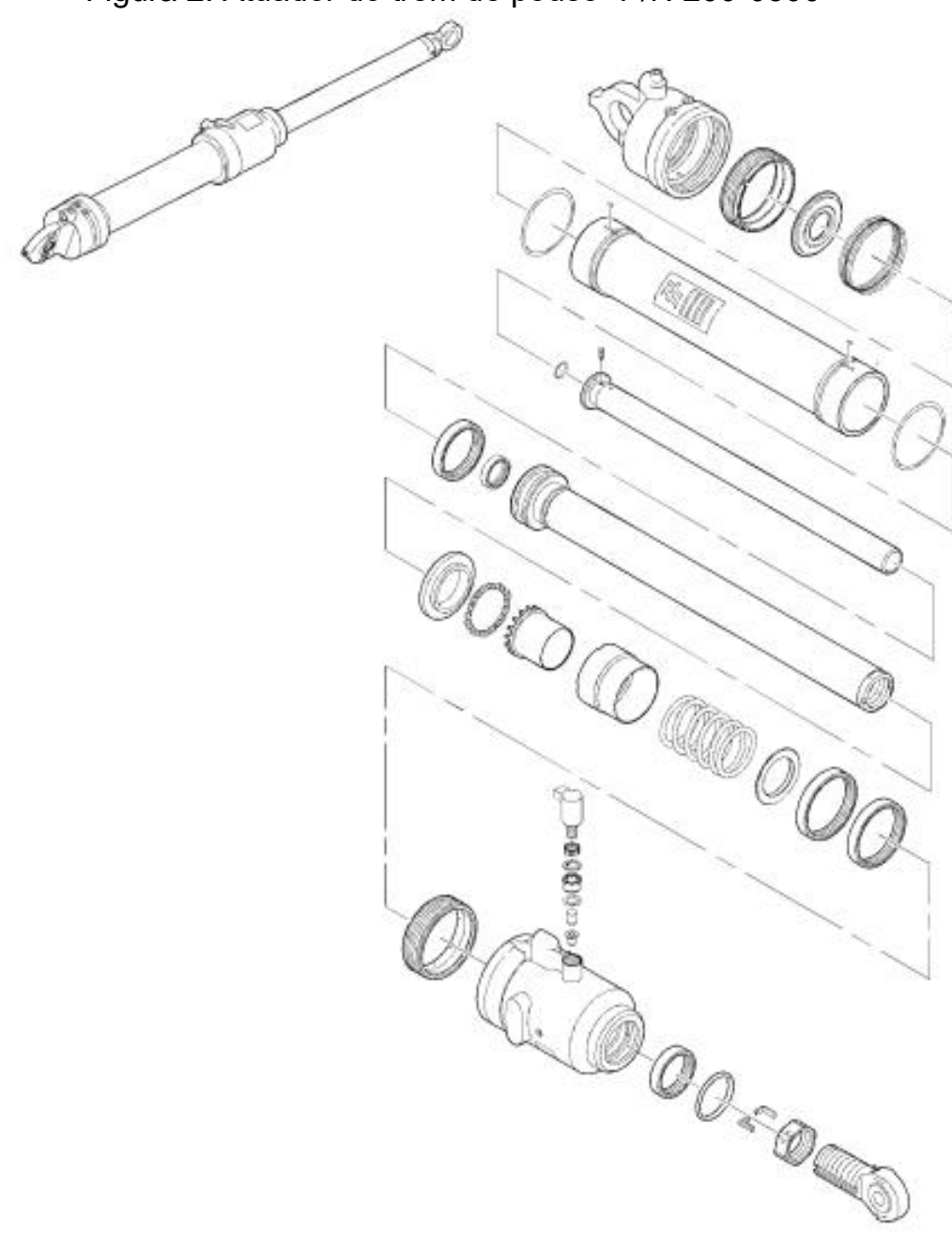

Fonte: Component Maintenance Manual (CMM) - Bombardier

\subsection{Desenvolvimento do aplicativo}

O desenvolvimento do aplicativo teve duas partes importantes: o modelamento das peças do atuador do trem de pouso e a programação das movimentações de montagem e desmontagem com a programação de um "painel" contendo as informações técnicas básicas do atuador de trem de pouso. Para o desenvolvimento de toda a programação das movimentações necessárias e para a visualização das informações técnicas do atuador, foi utilizado Unity com $C$-Sharp (C\#), uma linguagem rica de uso geral e de fácil aprendizado (UNITY, [s.d.]).

\section{Modelamento das Peças}

O modelamento de cada peça deveria ser em um grau de detalhes no qual a pessoa que fosse utilizar do aplicativo, reconhecesse qual peça estaria sendo manipulada, e que facilitaria a compreensão da ordem de montagem e desmontagem para a realização da manutenção do atuador. Considerando que esse nível de detalhes é relativamente alto, o software de desenvolvimento das peças teria que oferecer recursos de modelagem ampla, com opção de renderização para deixar as peças com visual mais realistas possíveis. $O$ software selecionado foi o Blender 2.8, que é uma suíte de criação 3D gratuita com código aberto. O Blender suporta a completude de modelação de pipeline-modelagem, simulação, 
rigging, renderização, composição e seguimento de movimento, entre outros recursos (BLENDER, [s.d.]).

Por conta das restrições implementadas durante a pandemia do COVID-19, não foi possível o modelamento de todas as peças do atuador do trem de pouso, sendo modeladas somente algumas peças, porém foram peças consideradas fundamentais para a compreensão total do funcionamento e a realização dos testes de treinamento de manutenção do atuador.

Figura 3: Peças modeladas no Blender

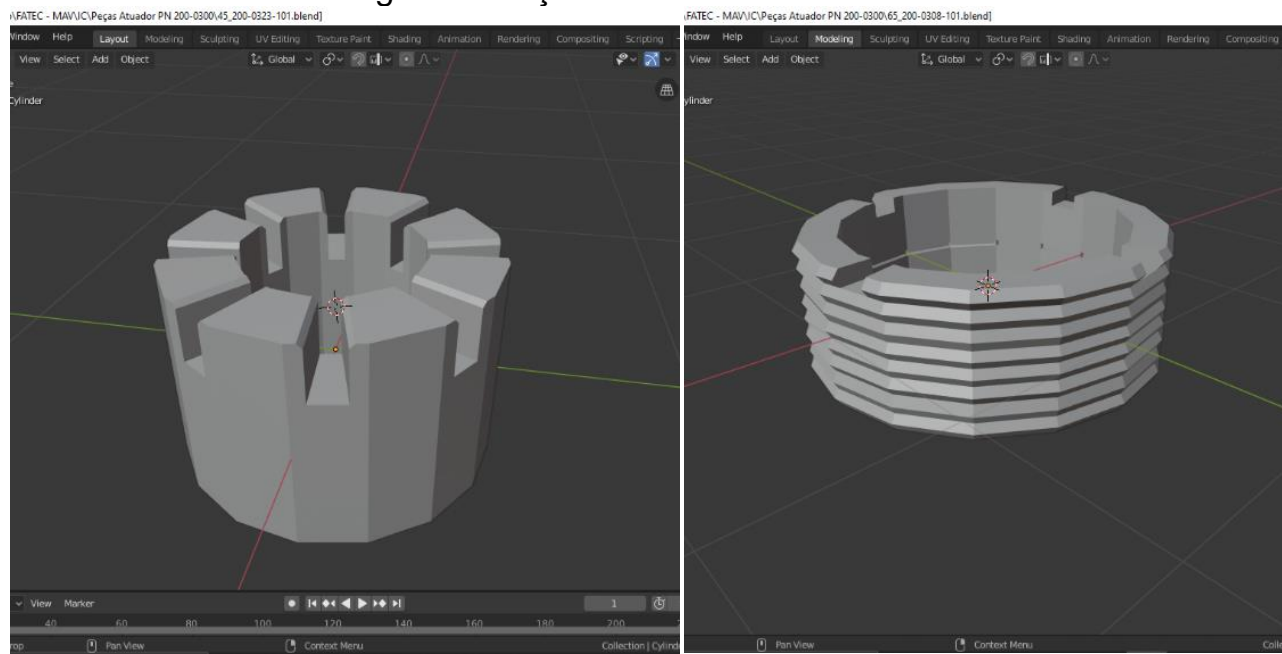

Fonte: Próprio Autor

\section{Programação Estrutural}

A configuração dos materiais na implementação atual das peças do atuador do trem de pouso contém cores fixas, mas na versão final do aplicativo as peças terão cores diferentes dependendo da posição em que elas se encontrarem, para a melhor visualização e compreensão de detalhes da montagem do atuador, como por exemplo, na posição inicial, as peças terão uma cor, na transição da posição inicial para a posição final elas terão uma segunda cor e na posição final elas terão uma terceira cor. A interface do aplicativo será simples e instrutiva, para que na sua utilização o usuário não encontre dificuldades.

\section{$4 \quad$ RESULTADOS}

O aplicativo foi desenvolvido para o sistema operacional Android, pois é o mais utilizado entre os alunos e professores do curso de manutenção aeroespacial. Ele contém as funções de montagem e desmontagem das peças do atuador do trem de pouso e informações técnicas básicas do atuador ao qual não é necessário o usuário sair do aplicativo para a realização da consulta dessas informações (Figura 4). Foi adicionado às suas configurações a detecção de um QR-Code, para que o aplicativo no momento de inicialização, não permitisse que as peças fossem inicializadas em uma localização muito diferente do usuário. 


\section{Evento Online}

Formação em Engenharia:

Tecnologia, Inovação e Sustentabilidade"

Figura 4: Informações técnicas básicas do atuador

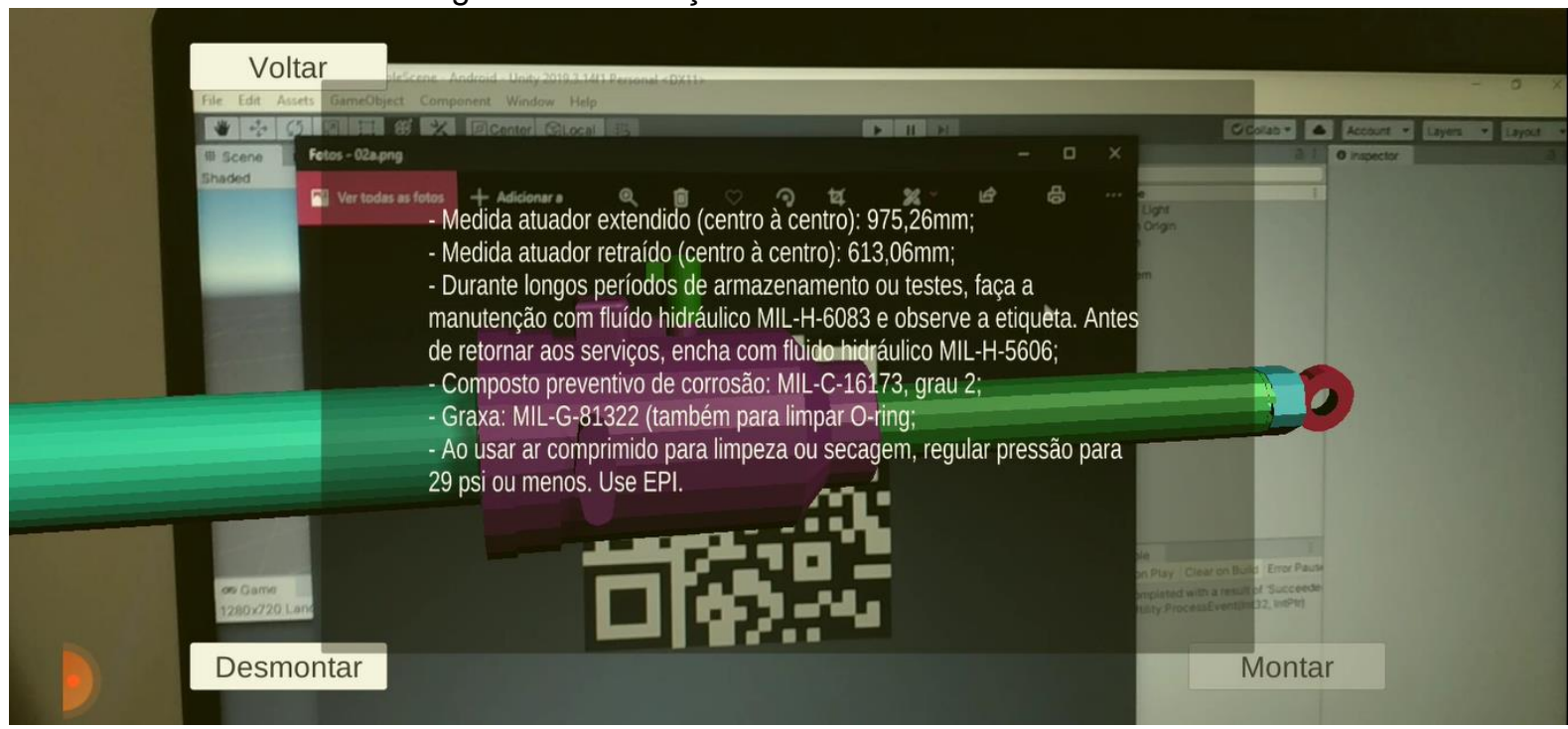

Fonte: Próprio Autor

A interface do aplicativo contém 3 botões touch: "Montar" para a realização gradual da montagem de cada peça; "Desmontar" para a realização gradual da desmontagem; "Infor." para ter acesso às informações do atuador.

As peças foram programadas para ficarem em uma espécie de armário imaginário (para uma melhor organização) (Figura 5), e conforme o botão de montar fosse sendo pressionado na tela do celular ou tablet, as peças vão montando (uma peça para cada clique do botão). Enquanto as peças estão na posição inicial ("armário") o botão de desmontagem ficaria desativado (Figura 5), pelo fato de não ter ainda nenhuma peça a ser desmontada. Ao final da montagem, o botão de montar desativa por não conter mais nenhuma peça a ser montada (Figura 6).

Figura 5: Peças em posição inicial (armário)

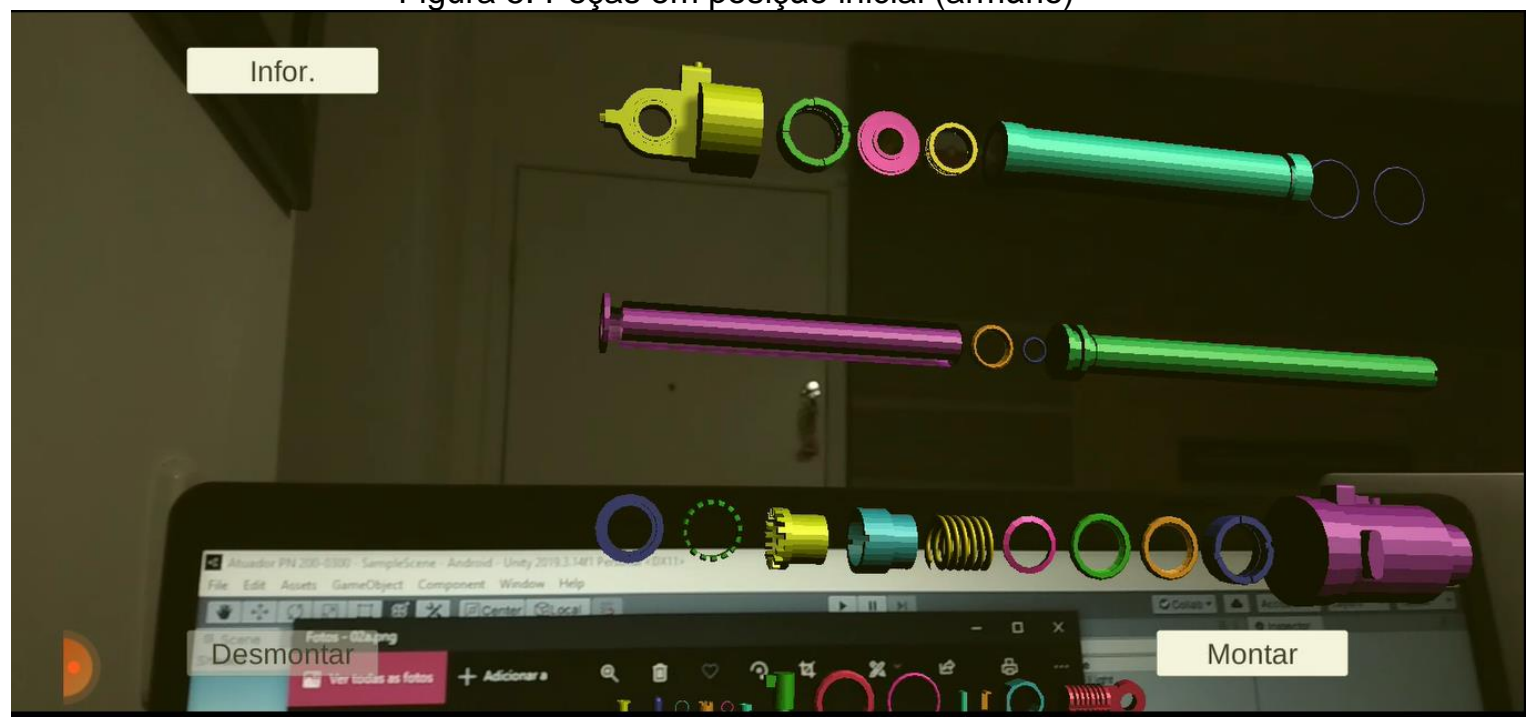

Fonte: Próprio Autor 


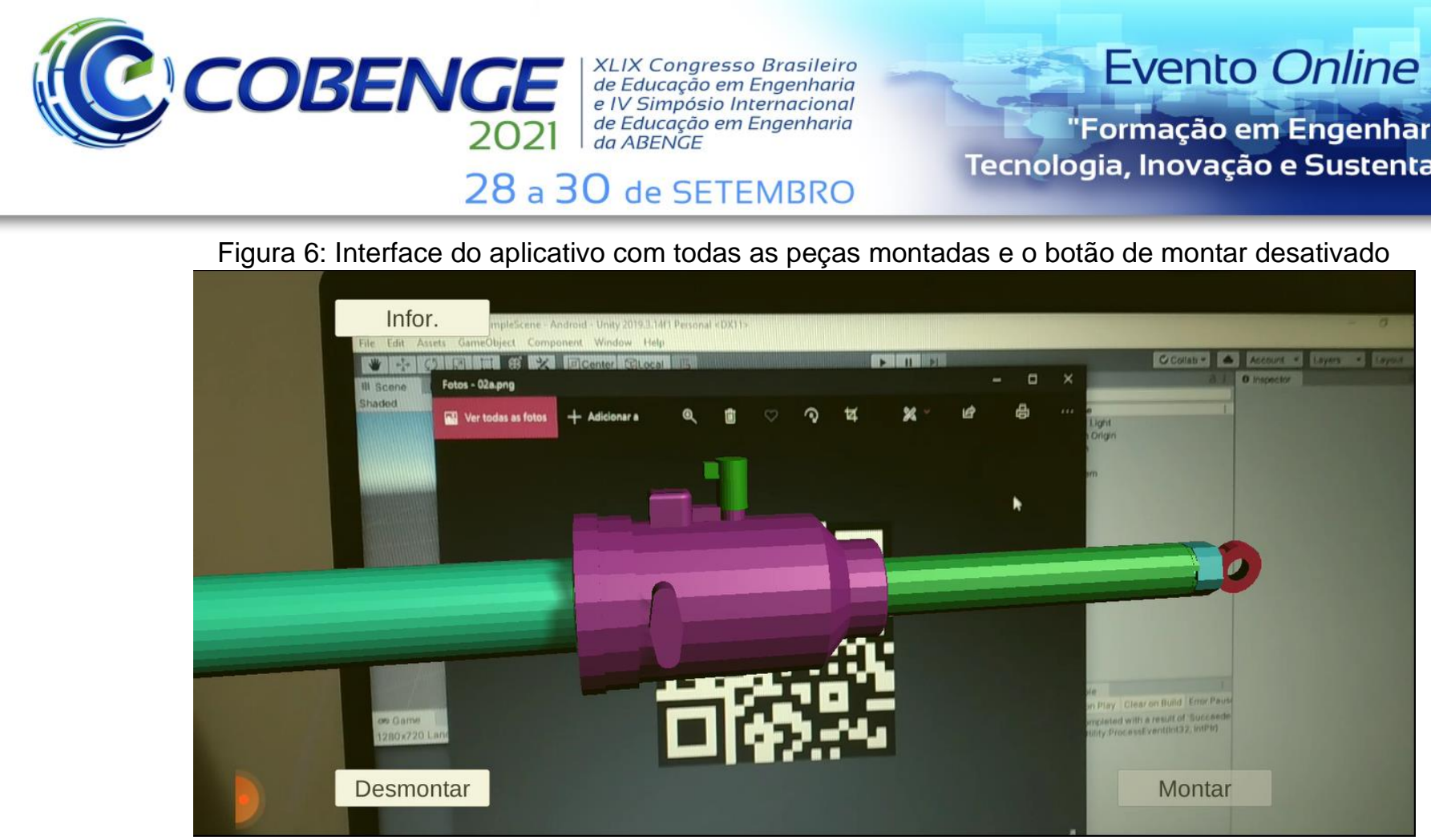

Fonte: Próprio Autor

As peças foram programadas em cores diferentes, para que a visualização da montagem fosse mais fácil e mais clara. Ao final da montagem, o atuador estará em posição de trabalho, ou seja, com o eixo totalmente avançado (Figura 7).

Figura 7: Atuador do trem de pouso em posição de trabalho

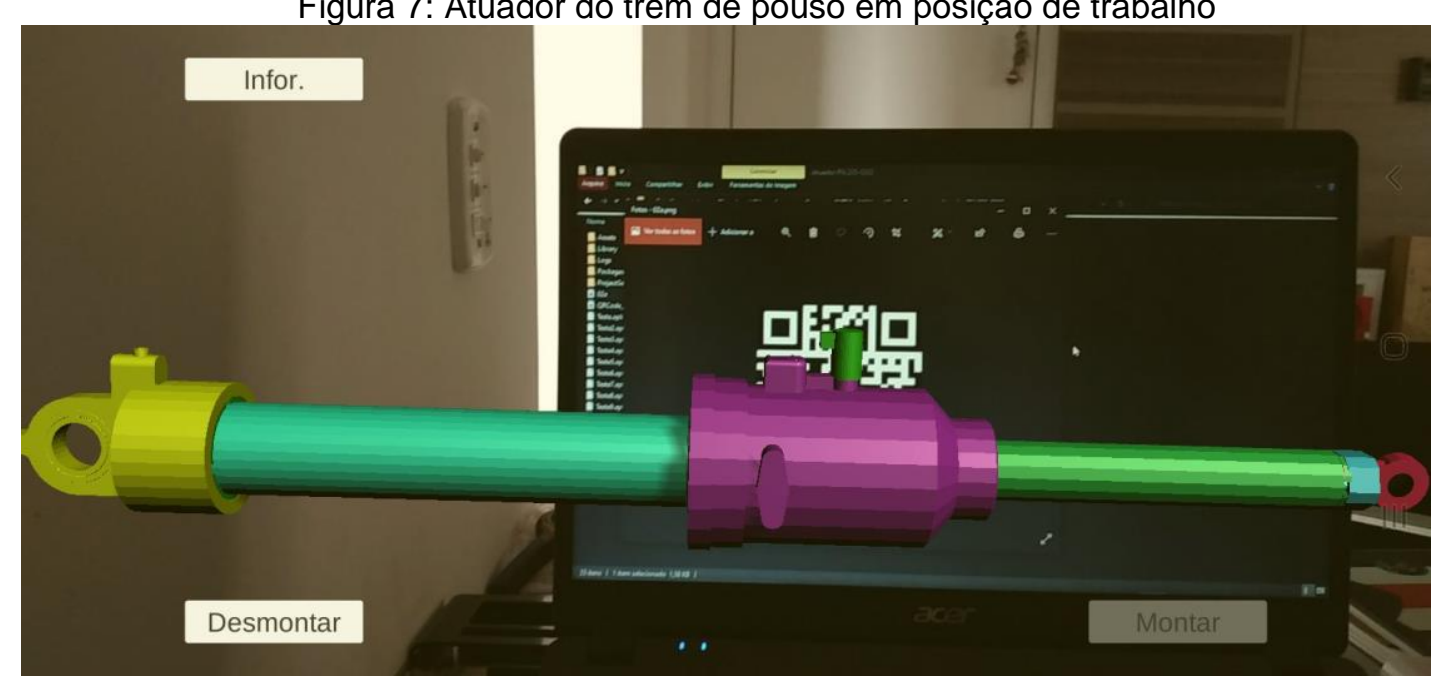

Fonte: Próprio Autor 
Este desenvolvimento demonstrou a possibilidade e viabilidade do desenvolvimento de ferramentas com código aberto e de fácil acesso, e principalmente a possibilidade de desenvolvimento com outros tipos de dispositivos, peças e ferramentas, para aprimorar mais esta ferramenta didática. Destaca-se que com o código aberto, o programa permite criação de novos algoritmos com outros exemplos de dispositivos, e permite melhorias e uma análise de solução de problemas mais complexos, pois a versão atual do aplicativo apresenta uma faixa restrita de problemas.

Testes do funcionamento das movimentações das peças e do uso da interface do aplicativo, serão realizados com os professores e alunos do curso de manutenção aeroespacial, para que sejam pontuadas melhorias e/ou para a devida aprovação da funcionalidade do aplicativo em sala de aula. Em razão do isolamento social causado pela pandemia do COVID-19, os testes do aplicativo com os professores e alunos do curso foram adiados. O aplicativo foi desenvolvido de uma maneira em que o aluno compreenderá mais claramente 0 assunto lecionado e o professor conseguirá avançar muito mais na abordagem de novos assuntos, cumprindo em menos tempo todo o roteiro de aprendizagem de sua matéria.

\section{Agradecimentos}

Os autores agradecem ao CNPq, pela oportunidade de desenvolver este projeto.

\section{REFERÊNCIAS}

AZUMA, R. T. A survey of augmented reality. In Presence: Teleoperators and Virtual Environments 6, 4, [S. I.], v. 6, 4, p. 355-385, 1997. Disponível em: https://doi.org/10.1561/1100000049. Acesso em: 05 ago. 2020.

BACCA, Jorge et al. Mobile Augmented Reality in Vocational Education and Training. Procedia Computer Science, 29 dez. 2015. Disponível em: https://doi.org/10.1016/j.procs.2015.12.203 . Acesso em: 11 ago. 2020.

BAZAROV, S. E.; KHOLODILIN, I. YU.; NESTEROV, A. S.; SOKHINA, A. V. Applying Augmented Reality in practical classes for engineering students. In: IOP Conference Series: Earth and Environmental Science, 2017, Chelyabinsk. IPDME. Chelyabinsk. Dlsponível em: https://iopscience.iop.org/article/10.1088/1755-1315/87/3/032004. Acesso em: 16 out. 2020 .

BILLINGHURST, M.; KATO, H. Collaborative Mixed Reality. In: Proceedings of the First International Symposium on Mixed Reality (ISMR), 1999. Mixed Reality - Merging Real and Virtual Worlds, pp. 261-284. Berlin: Springer Verlag. Disponível em: https://www.vs.inf.ethz.ch/edu/SS2005/DS/papers/ar/billinghurst-ar.pdf . Acesso em: 04 ago.2021.

BLENDER. Disponível em: https://www.blender.org/. Acesso em: 04 out. 2020.

BOEING. Boeing tests Augmented Reality in the factory. Disponível em: https://www.boeing.com/features/2018/01/augmented-reality-01-18.page . Acesso em: 18 jul. 2020. 
CABERO-ALMENARA, Julio; FERNÁNDEZ-BATANERO, José M.; BARROSO-OSUNA, Julio. Adoption of augmented reality technology by university students. Heliyon, Seville, 14 mai. 2019. Disponível em: https://doi.org/10.1016/j.heliyon.2019.e01597 . Acesso em: 27 dez. 2020.

DADALD, Eduardo Pares. Aplicação de realidade aumentada no ensino.2015.

Trabalho de conclusão de curso - Curso de Design Visual. Universidade Federal do Rio Grande do Sul, Porto Alegre, 2015. Disponível em:

https://www.lume.ufrgs.br/bitstream/handle/10183/141000/000991613.pdf?sequence=1 .

Acesso em: 18 jul. 2020.

DOMINGOS, D. d. et al. A Realidade Aumentada como objeto de aprendizagem: as novas tecnologias na EAD. In: XII Colóquio Internacional de Gestión Universitária, 2012, Veracruz. Anais. Veracruz. Disponível em:

http://repositorio.ufsc.br/xmlui/handle/123456789/97679. Acessado em: 16 jul. 2020.

JÚNIOR, David Severo do Nascimento. Realidade Aumentada na Educação: Uma análise das ferramentas Flaras e Aumentaty como recursos para aulas expositivas. 2017. Trabalho de Conclusão de Curso - Licenciatura em Computação. Universidade Federal da Paraíba, João Pessoa, 2017. Disponível em:

https://seer.ufrgs.br/renote/article/view/86010 . Acesso em: 10 mar. 2021.

KAWAKAME, Eric Kenzo Tsuha; MARTINS, Igor Ferreira. Análise simplificada de um trem de pouso em elementos finitos. 2016. Trabalho de Conclusão de Curso - Curso de Engenharia Mecânica. Universidade Federal Fluminense, Niterói, Rio de Janeiro, 2016. Disponível em:

https://app.uff.br/riuff/bitstream/1/3594/1/An\%C3\%A1lise\%20Simplificada\%20de\%20um\% 20Trem\%20de\%20Pouso\%20em\%20Elementos\%20Finitos\%20-

$\% 20 \% 20$ Eric\%20Kenzo\%20e\%20Igor\%20Martins\%20\%202016.2\%20Proj.pdf . Acesso em: 18 jul. 2020.

KIRNER, Claudio; SISCOUTTO, Robson. Realidade virtual e aumentada: conceitos, projeto e aplicações. Petrópolis: Editora SBC, 2007. p. 4-6.

MADANIPOUR, Parian; COHRSSEN, Caroline. Augmented reality as a form of digital technology in Early childhood education. Australasian Journal of Early Childhood, Melbourne, 20 nov. 2019. Disponível em:

https://journals.sagepub.com/doi/full/10.1177/1836939119885311. Acesso em: 07 jan. 2021.

PETROV, Plamen D.; ATANASOVA, Tatiana V. The Effect of Augmented Reality on Students' Learning Performance in Stem Education. Information, Sófia, 15 abr. 2020. Disponível em: https://www.mdpi.com/2078-2489/11/4/209. Acesso em: 07 jan. 2021.

POLITECNICO DI MILANO. Landing Gear System. Milão: Dipartmento di Ingeneria Aerospaziale Aircraft Systems, 2004.

SILVA, D.; COSTA, J.; INGRACIO, P; OLIVEIRA, W. Realidade Virtual Aumentada Aplicada como Ferramenta de Apoio ao Ensino. Revista Tecnologias em Projeção, Brasília, v.2, n.1, p. 11-15, 2011. 
UNITY. Disponível em: https://unity.com/pt. Acesso em: 16 jun. 2020.

UNITY. Disponível em: https://unity.com/pt/how-to/beginner-game-coding-resources. Acesso em: 22 jun. 2020.

\title{
DEVELOPMENT OF AN AUGMENTED REALITY APPLICATION FOR APPLY IN THE AEROSPACE MAINTENANCE COURSE
}

\begin{abstract}
Augmented reality (AR) technology has been in high demand and is the target of research due to its ease of use and the real-time interaction that exists between the virtual world and its user. With the use of three-dimensional models (by means of a smartphone or tablet) being superimposed on real environments, augmented reality is well evaluated and suitable to be applied in the educational sector, helping more and more the teaching-learning process, increasing more the interest and learning of the students. This article consists in the presentation of the development of an application compatible with the Android operating system (most usual among students and teachers), helping in aerospace maintenance classes both in classroom and remote classes, having as object of study a landing gear actuator, and can be used in: explanation of assembly and disassembly of the actuator; demonstration of preventive and corrective maintenance; exercise application and extraclass.
\end{abstract}

Keywords: Augmented reality. Engineering. Education. Maintenance. Aircraft. 